\title{
Carbon Ion Radiotherapy for Locally Recurrent Rectal Cancer
}

\author{
Sonal S. Noticewala, MD, MAS, and Prajnan Das, MD, MS, MPH, FACR, FASTRO \\ Division of Radiation Oncology, The University of Texas MD Anderson Cancer Center, Houston, TX
}

Patients with rectal cancer initially treated with short- or long-course radiotherapy followed by total mesorectal excision have a pelvic recurrence rate of about $5-10 \% .^{1-5}$ Thus, theoretically, those treated with prior radiation may likely benefit from potentially higher doses of radiation in the setting of pelvic recurrence. Compared with X-ray (photon) radiotherapy, particle radiotherapy has unique physical and biologic properties that allow delivery of higher doses of radiation to the tumor with more normal tissue sparing. While proton radiotherapy has been the mainstay in particle radiotherapy, carbon ion radiotherapy (CIRT) could be even more advantageous. ${ }^{6}$ CIRT causes less lateral radiation scatter, leading to better radiation dose distribution and improved organ sparing. It also produces more dense ionizations, leading to higher relative biological effectiveness (RBE), along with reduced effect of hypoxia, lower deoxyribonucleic acid (DNA) damage repair, and less cell cycle dependence, potentially leading to greater effects on the tumor. ${ }^{7}$ Thus, there has been growing interest in leveraging the physical and radiobiologic properties of CIRT in the setting of re-irradiation of various disease sites such as in abdominal and pelvic malignancies.

In this issue of Annals of Surgical Oncology, Yamada et al. ${ }^{8}$ retrospectively reported on the safety and efficacy of CIRT in the setting of locally recurrent rectal cancer in patients with prior pelvic irradiation. In that study, 77 patients with locally recurrent rectal cancer who had previously received a median dose of $50 \mathrm{~Gy}$ were treated with CIRT to 70.4 Gy (RBE) in 16 fractions over 4 weeks. The

(C) Society of Surgical Oncology 2021

First Received: 21 September 2021

Accepted: 24 September 2021;

Published Online: 6 October 2021

P. Das, MD, MS, MPH, FACR, FASTRO

e-mail: prajdas@mdanderson.org overall 3- and 5-year LC rates were $69 \%$ and $62 \%$, respectively, and the overall 3- and 5-year regional control (RC) rates were $85 \%$ and $81 \%$, respectively. The median overall survival was 47 months. The authors also reported that $8(10 \%)$ patients developed acute grade 3 toxicity, of whom 5 were grade 3 pelvic infections. Late grade 3 toxicities occurred in $16(21 \%)$ patients, which included 13 late grade 3 pelvic infections. The authors concluded that re-irradiation with CIRT for locally recurrent rectal cancer is safe and effective with acceptable toxicity.

CIRT has been previously investigated in abdominal and pelvic malignancies. In the setting of recurrent rectal cancer, a dose escalation phase I trial showed 5-year local control and survival rates of $88 \%$ and $59 \%$, respectively, with no acute reactions higher than grade 3 in patients treated at the highest dose level of $73.6 \mathrm{~Gy}$ (RBE). ${ }^{9}$ In addition to recurrent rectal cancer, CIRT has been evaluated and extensively reviewed in the setting of pancreatic cancer. ${ }^{10}$ The efficacy of radiotherapy in pancreatic cancer is often limited by its inherent radioresistance likely secondary to hypoxia ${ }^{11}$ and the proximity to surrounding normal tissue such as the duodenum. However, several studies have shown CIRT to be a promising treatment option to improve overall survival and local control with limited toxicity in patients with resectable ${ }^{12}$ and locally advanced ${ }^{13}$ pancreatic cancer.

Prior studies on X-ray (photon) re-irradiation for recurrent rectal cancer have reported accelerated hyperfractionated approaches involving twice-daily treatment. ${ }^{14,15}$ Valentini et al. reported a phase II trial of 40.8 Gy in 1.2 Gy twice-daily fractions in 59 patients, with 5 -year local control of $38.8 \%$ in all patients and $69.0 \%$ in radically resected patients. ${ }^{14}$ Similarly, Tao et al. reported that a median dose of $39 \mathrm{~Gy}$ in $1.5 \mathrm{~Gy}$ twice-daily fractions in 102 patients resulted in 3-year freedom from local progression of $40 \%$ in all patients and $49 \%$ in patients undergoing surgical resection. ${ }^{15}$ In contrast, Yamada et al. report 3- and 5-year LC rates of $69 \%$ and $62 \%$, 
respectively, which appear higher compared with that in the prior studies, especially given that patients in the study by Yamada et al. did not undergo surgical resection. Acute and late toxicity from CIRT also appears comparable with what has been previously reported in patients with prior radiation receiving X-ray radiotherapy for recurrent rectal cancer.

Overall, Yamada et al. add to the growing body of literature regarding the use of CIRT in previously irradiated malignancies. However, it is important to note that the patients treated in this cohort were carefully selected. For example, the recurrent tumor edge had to be greater than $3 \mathrm{~mm}$ from key structures (bowel, bladder, and urethra); these criteria would exclude many common sites of recurrences, including anastomotic recurrences. Therefore, CIRT would be a viable option only for a subset of patients with locally recurrent rectal cancer. Such selection criteria also make it difficult to compare the results with that in prior studies using other radiation modalities. Furthermore, it is important to note that CIRT has very limited availability, with only 13 centers worldwide. Further studies would be needed to determine if CIRT is a cost-effective option at the patient, provider, and healthcare system level.

In conclusion, Yamada et al. demonstrate that CIRT is a promising radiation modality that provides durable LC control rates with acceptable rates of toxicity in select patients with locally recurrent rectal cancer treated with prior pelvic irradiation. Future studies should aim to further evaluate the role of CIRT for recurrent cancers, ideally through randomized comparisons with other radiation approaches.

FUNDING No sources of funding were received to assist in the preparation of this editorial.

DISCLOSURE Sonal S. Noticewala has no conflicts of interest to declare. Prajnan Das has received honorarium from the American Society for Radiation Oncology and the National Cancer Institute.

\section{REFERENCES}

1. Das P, Skibber JM, Rodriguez-Bigas MA, et al. Clinical and pathologic predictors of locoregional recurrence, distant metastasis, and overall survival in patients treated with chemoradiation and mesorectal excision for rectal cancer. Am J Clin Oncol. 2006;29(3):219-24.
2. Sauer R, Becker H, Hohenberger W, et al. Preoperative versus postoperative chemoradiotherapy for rectal cancer. $N$ Engl J Med. 2004;351(17):1731-40.

3. Bujko K, Nowacki MP, Nasierowska-Guttmejer A, et al. Longterm results of a randomized trial comparing preoperative shortcourse radiotherapy with preoperative conventionally fractionated chemoradiation for rectal cancer. $B r \quad J$ Surg. 2006;93(10):1215-23.

4. Erlandsson J, Holm T, Pettersson D, et al. Optimal fractionation of preoperative radiotherapy and timing to surgery for rectal cancer (Stockholm III): a multicentre, randomised, non-blinded, phase 3, non-inferiority trial. Lancet Oncol. 2017;18(3):336-46.

5. Ngan SY, Burmeister B, Fisher RJ, et al. Randomized trial of short-course radiotherapy versus long-course chemoradiation comparing rates of local recurrence in patients with $\mathrm{T} 3$ rectal cancer: trans-tasman radiation oncology group trial 01.04. J Clin Oncol. 2012;30(31):3827-33.

6. Kamada T, Tsujii H, Blakely EA, et al. Carbon ion radiotherapy in Japan: an assessment of 20 years of clinical experience. Lancet Oncol. 2015;16(2):e93-100.

7. Ohno T. Particle radiotherapy with carbon ion beams. EPMA J. 2013;4(1):9.

8. Yamada S, Takiyama H, Isozaki Y, et al. Carbon ion radiotherapy for locally recurrent rectal cancer in patients with prior pelvic irradiation. Ann Surg Oncol. 2021. https://doi.org/10.1245/s104 34-021-10876-4.

9. Yamada S, Kamada T, Ebner DK, et al. Carbon-ion radiation therapy for pelvic recurrence of rectal cancer. Int J Radiat Oncol Biol Phys. 2016;96(1):93-101.

10. Liermann J, Shinoto M, Syed M, et al. Carbon ion radiotherapy in pancreatic cancer: a review of clinical data. Radiother Oncol. 2020;147:145-50.

11. Koong AC, Mehta VK, Le QT, et al. Pancreatic tumors show high levels of hypoxia. Int J Radiat Oncol. 2000;48(4):919-22.

12. Shinoto M, Yamada S, Yasuda S, et al. Phase 1 trial of preoperative, short-course carbon-ion radiotherapy for patients with resectable pancreatic cancer. Cancer. 2013;119(1):45-51.

13. Kawashiro S, Yamada S, Okamoto M, et al. Multi-institutional study of carbon-ion radiotherapy for locally advanced pancreatic cancer: japan carbon-ion radiation oncology study group (JCROS) study 1403 pancreas. Int J Radiat Oncol Biol Phys. 2018;101(5):1212-21.

14. Valentini V, Morganti AG, Gambacorta MA, et al. Preoperative hyperfractionated chemoradiation for locally recurrent rectal cancer in patients previously irradiated to the pelvis: a multicentric phase II study. Int $J$ Radiat Oncol Biol Phys. 2006;64(4):1129-39.

15. Tao R, Tsai CJ, Jensen G, et al. Hyperfractionated accelerated reirradiation for rectal cancer: an analysis of outcomes and toxicity. Radiother Oncol. 2017;122(1):146-51.

Publisher's Note Springer Nature remains neutral with regard to jurisdictional claims in published maps and institutional affiliations. 